\title{
The Study to Understand Mortality and Morbidity in COPD (SUMMIT) study protocol
}

\author{
Jørgen Vestbo, Julie Anderson, Robert D. Brook, Peter M.A. Calverley, Bartolome \\ R. Celli, Courtney Crim, Brett Haumann, Fernando J. Martinez, Julie Yates and David \\ E. Newby
}

ABSTRACT: Chronic obstructive pulmonary disease (COPD) often coexists with other chronic diseases and comorbidities that can markedly influence patients' health status and prognosis. This is particularly true for cardiovascular disease (CVD). However, there have been no trials assessing the effect of COPD medications on CVD in patients with both diseases.

The "Study to Understand Mortality and Morbidity in COPD" (SUMMIT) aims at determining the impact of fluticasone furoate/vilanterol combination and the individual components on the survival of patients with moderate COPD and either a history of CVD or at increased risk for CVD.

SUMMIT is a multicentre, randomised, double-blind, parallel-group, placebo-controlled trial of 16000 patients with moderate COPD randomly assigned to once daily treatment with fluticasone furoate/vilanterol $(100 / 25 \mu \mathrm{g})$, fluticasone furoate $(100 \mu \mathrm{g})$, vilanterol $(25 \mu \mathrm{g})$ or matched placebo; mortality is the primary end-point. The study is an event-driven trial powered by the comparison of furoate/vilanterol versus placebo. Secondary end-points are decline in forced expiratory volume in $1 \mathrm{~s}$ and effect on a composite cardiovascular end-point. This article describes the design of the SUMMIT study.

KEYWORDS: Cardiovascular disease, chronic obstructive pulmonary disease, mortality

$\mathbf{T}$ he morbidity and mortality of chronic obstructive pulmonary disease (COPD) continues to increase [1]. It is increasingly recognised that COPD often coexists with other chronic diseases and that comorbidities can contribute to patients' health status and prognosis $[2,3]$. Some of the changes seen in patients with COPD have been termed extrapulmonary manifestations of COPD and typically include features that are believed to be linked to progression of COPD such as skeletal muscle dysfunction [4], lean mass depletion [5], and osteoporosis and osteopenia [6].

Other comorbidities are not so obviously linked to disease progression and these include cardiovascular disease (CVD). Several prospective studies have reported an association between impaired pulmonary function and cardiovascular morbidity and mortality [7-10] even after adjusting for accepted CVD risk factors. Epidemiological data suggest that patients with COPD are at a greater risk of CVD compared with age- and sex-matched controls without COPD [11]. Furthermore, more patients with mild-to-moderate COPD die from lung cancer and CVDs, such as coronary artery disease and stroke, than from the respiratory effects of COPD [12-14]. Systemic inflammation has been proposed as having a potential role in explaining the association between COPD and increased risk of CVD [15]. Other factors have also been implicated, including autonomic imbalance, vascular endothelial dysfunction, lower arterial compliance and arrhythmias. It is, however, also possible that the decreased physical activity associated with even mild COPD [16] may increase the risk of CVD, as well as other comorbidities.

The current Global Initiative for Obstructive Lung Diseases (GOLD) strategy document has highlighted the need to assess and treat comorbidities in COPD [1]. For most CVDs, the advice is to treat the comorbidity as if the patient did not have COPD and to treat COPD as in patients
AFFILIATIONS

For a list of the author's affiliations, see the Acknowledgements.

CORRESPONDENCE

J. Vestbo

Dept of Respiratory Medicine J

Odense University Hospital

Sdr Ringvej 29

5000 Odense C

Denmark

E-mail: jvestbo@dadlnet.dk

Received:

June 022012

Accepted after revision:

Aug 012012

First published online:

Sep 272012 
without CVD. However, the evidence base for treating COPD patients with comorbidities is weak and most advice comes from expert statements or from secondary analyses of large studies. An example of the latter is the secondary analysis of the TORCH (Towards a Revolution in COPD Health) study, which focused on the safety of COPD medications in patients with concomitant CVD entered into this large 3-year study [17]. In addition to providing confidence in the safety of treating these patients with salmeterol alone or in combination with fluticasone propionate, the study also indicated that treatment with combined salmeterol and fluticasone propionate could have beneficial effects on the risk of cardiovascular adverse events. Similarly, combination treatment seemed to have a similar effect on respiratory and cardiovascular mortality in the primary TORCH report [14]. Therefore, it seems reasonable to hypothesise that combination treatment may have a beneficial effect on survival in patients with COPD and concomitant CVD.

The Study to Understand Mortality and Morbidity in COPD (SUMMIT) is designed to prospectively compare the efficacy of fluticasone furoate/vilanterol in combination $(100 / 25 \mu \mathrm{g})$, fluticasone furoate alone $(100 \mu \mathrm{g})$ or vilanterol alone $(25 \mu \mathrm{g})$ against matched placebo once daily via the novel dry powder inhaler on survival in subjects with moderate COPD and a history of, or at increased risk for, CVD. The study will test the hypothesis that treatment with combined inhaled corticosteroids (ICS) and long-acting $\beta$-agonists will reduce mortality when compared with placebo. As most patients with severe or very severe airflow limitation will require treatment with longacting bronchodilators and possibly ICS, and because comorbidities seem independent of severity of airflow limitation [2-5], the study will include patients with moderate airflow limitation only. This article describes the protocol design and the approaches taken in this study that we anticipate will have important implications for the future treatment of patients with COPD.

\section{METHODS}

\section{SUMMIT study design}

This is an international, multicentre, placebo-controlled, double-blind, randomised, parallel-group trial, with patients expected to contribute 15-44 months of study time. The study involves a 4-10 day run-in period, a treatment phase and a 1week follow-up phase. The length of the treatment phase will depend on the mortality rate in the study; the study will last until 1000 deaths have been recorded. All prior use of ICS and inhaled long-acting bronchodilators will be discontinued at entry to the run-in period. The population sought are those patients with COPD that the practitioner believes can be adequately managed without these medications. Patients will be treated on an outpatient basis and clinic visits will occur at screening, randomisation, 4 weeks, and then every 12 weeks until the study has reached the required number of events.

\section{Interventions}

A total of 16000 patients with moderate COPD and a history of, or at increased risk for, CVD will be randomised from $\sim 1100$ sites to one of the following four treatment groups: placebo; once-daily ICS fluticasone furoate $(100 \mu \mathrm{g})$; once daily inhaled $\beta_{2}$-agonist vilanterol $(25 \mu \mathrm{g})$; or fluticasone furoate/ vilanterol in combination $(100 / 25 \mu \mathrm{g})$, administered once daily via the novel dry powder inhaler. A separate randomisation schedule will be produced for each country. Patients who withdraw prematurely from study treatment will still be followed up through regular contact until study termination to determine survival status. The study is listed on ClinicalTrials.gov (www. clinicaltrials.gov identifier NCT01313676).

\section{Organisational committees}

The study is being guided by a Steering Committee consisting of external clinical experts and representatives of GlaxoSmithKline (London, UK). An independent Safety and Efficacy Data Monitoring Committee is overseeing the ethical and safety interests of the patients by periodically reviewing results of interim analyses and cumulative data on serious adverse events. A Clinical End-point Committee is independently reviewing and categorising the cause of death, as well as events that are part of the cardiovascular composite end-point for each patient where an event has been recorded.

\section{Patient participation}

Recruitment commenced in March 2011 and is expected to conclude in late 2013. Patients are aged 40-80 years with a smoking history of $\geqslant 10$ pack-years, a clinical diagnosis of COPD with forced expiratory volume in $1 \mathrm{~s}$ (FEV1)/forced vital capacity $<0.70$ and moderate airflow limitation, defined as a post-albuterol/salbutamol FEV1 $\geqslant 50$ and $\leqslant 70 \%$ of predicted normal values, calculated using National Health and Nutrition Examination Survey III reference equations $[18,19]$. In addition, patients are required to have a history of CVD or to be at increased risk for CVD. The inclusion and most important exclusion criteria are summarised in table 1.

Patients who have an exacerbation of COPD during the run-in period that requires systemic corticosteroid therapy or hospitalisation will not be eligible for randomisation. Patients are allowed to take COPD medication except for ICS, longacting bronchodilators and long-term oral corticosteroids. Tiotropium use will not be permitted at baseline. However, if during the double-blind treatment phase, a subject experiences a severe COPD exacerbation (i.e. requiring hospitalisation) and requires additional treatment or experiences multiple moderate exacerbations, tiotropium may be added. Tiotropium can also be added by the investigator following a discussion with the study medical monitor if the investigator believes the addition is warranted to improve the management of the subject's disease. All patients will be offered salbutamol as relief medication.

The study is conducted in accordance with the Declaration of Helsinki and Good Clinical Practice Guidelines. All patients will have given written informed consent before participating in the study. Ethics and Review Boards of all participating institutions have approved this protocol.

\section{Efficacy and health outcome assessments}

The primary end-point measure will be all-cause mortality. Secondary end-points and other objectives are given in table 2. An important secondary end-point is the cardiovascular composite end-point comprising of on-treatment cardiovascular death, myocardial infarction, stroke, unstable angina and transient ischaemic attack. 


\section{TABLE 1 Inclusion and exclusion criteria for the SUMMIT study}

\section{Key inclusion criteria}

Male or female aged $40-80$ years

Current or ex-smokers with a smoking history of $\geqslant 10$ pack-years

An established history of COPD with an FEV $1 / F V C$ ratio $<0.70$ and an $\mathrm{FEV}_{1} \geqslant 50$ and $\leqslant 70 \%$ of predicted normal

A history of CVD or to be at increased risk for CVD

For patients $\geqslant 40$ years of age this is defined as any one of the following: established coronary artery disease, established peripheral vascular disease, previous stroke, previous myocardial infarction or diabetes mellitus with target organ disease

For patients $\geqslant 60$ years of age, any one of the above or two of the following: being treated for hypercholesterolaemia, being treated for hypertension, being treated for diabetes mellitus or being treated for peripheral vascular disease

\section{Key exclusion criteria}

Current diagnosis of asthma or respiratory disorders other than COPD

Chest radiograph indicating diagnosis other than COPD

Undergone lung volume reduction surgery and/or a lung transplant

Requirement for long-term oxygen therapy at start of study ( $>12 \mathrm{~h}$ per day)

Receiving long-term oral corticosteroid therapy

Current severe heart failure (NYHA class IV), subjects will also be excluded if they have a known ejection fraction of $<30 \%$ or if they have an implantable cardioverter defibrillator

Any life-threatening condition with life expectancy $<3$ years, other than vascular disease or COPD, that might prevent the subject from completing the study End-stage chronic renal disease

COPD: chronic obstructive pulmonary disease; SUMMIT: Study to Understand Mortality and Morbidity in COPD; FEV1: forced expiratory volume in 1 s; FVC: forced vital capacity; CVD: cardiovascular disease; NYHA: New York Heart Association.

\section{Survival status}

Survival status of each subject will be recorded at every visit. For any subject who prematurely withdraws, survival status will be captured at 3-monthly intervals by means of telephone calls or other forms of contact. Accurate assignment of cause of death is essential. The investigator will assign a cause of death based on contact with the attending physician (where possible), details given on the death certificate, autopsy findings (if any) and any other available clinical evidence. Categorisation of cause of death will also be performed centrally by a Clinical End-point Committee who will review study data and any additional information available (e.g. details given on the death certificate, autopsy findings, and any other available clinical evidence). The categorisation of cause of death assigned by the Clinical
End-point Committee will be the primary basis for all analyses for specific cause of death. The Clinical End-point Committee will build on decision rules developed in the TORCH study [20].

\section{Clinical safety assessments}

Each adverse event or concurrent illness during the study will be documented on the case report form. For the purpose of this study, a moderate COPD exacerbation is defined as an exacerbation treated with antibiotics and/or systemic corticosteroids whereas a severe COPD exacerbation required hospitalisation. In this study, pneumonia is defined as new auscultatory findings compatible with parenchymal lung infection and/or radiographic evidence of parenchymal/air space disease. A confirmed diagnosis of pneumonia must be

\section{TABLE 2 Secondary end-points and other objectives in the SUMMIT study}

\section{Secondary end-points}

To evaluate the effect of FF/VI compared with placebo on the rate of decline in FEV1

To evaluate the effect of FFNI compared with placebo on a cardiovascular composite end-point comprised of on-treatment cardiovascular death, myocardial infarction stroke, unstable angina and TIA

\section{Other objectives}

To evaluate the following treatment comparisons on all primary, secondary, exploratory and other end-points: FF/ compared with FF, FF/ VI compared with VI, FF compared with placebo, and VI compared with placebo

To evaluate the effect of FFNI compared with placebo on the rate of moderate/severe COPD exacerbations

To evaluate the effect of FFNI compared with placebo on COPD-related mortality

To evaluate the effect of FFNI compared with placebo on arterial stiffness in a subset of subjects

To evaluate the effect of FFNI compared with placebo on health-related quality of life measured with the SGRQ-C in a subset of subjects

To evaluate quality-adjusted life years by treatment group using health status data collected from EuroQol Questionnaire in a subset of subjects

To evaluate the impact of FFNI compared with placebo on healthcare resource utilisation (measured by number of days hospitalised for COPD) 
recorded as an adverse event. Patients with worsening COPD status or progressive CVD while on study treatment can receive other medications, or be withdrawn if in the investigator's opinion the patient's deterioration prevents ongoing participation. The reason for withdrawal will be recorded and patients will be followed up until study termination.

One formal interim analysis of the time to death from any cause is planned in addition to the final analysis. It is intended that this analysis will be performed when $\sim 50 \%$ of the total deaths have been observed. The analysis method for this will be the same as that used in the final analysis, i.e. Cox proportional hazards model.

\section{Statistical analysis}

This event driven study is designed to have $90 \%$ power to detect a 30\% reduction in the risk of all-cause mortality (hazard ratio 0.70 ) on fluticasone furoate/vilanterol compared with placebo at the two-sided $1 \%$ significance level. In order to detect this reduction, 478 events (on fluticasone furoate/ vilanterol and placebo combined) would be required.

The effects of the components (fluticasone furoate and vilanterol) are expected to be lower than for fluticasone furoate/ vilanterol. The study is not powered for comparisons of the components to placebo or for the combination to components. If the true mortality rates for the components are intermediate between fluticasone furoate/vilanterol and placebo, then it would be expected that by the time there are 478 deaths between the fluticasone furoate/vilanterol and placebo arms there should be a roughly similar number on the component arms combined, which would give a total of 956 on four arms. However, since the study is blinded and it will be unknown exactly how many deaths will have occurred on the fluticasone furoate/vilanterol and placebo arms when it stops, a balance must be struck between ensuring it runs long enough to accrue 478 deaths on those arms versus continuing the study for longer than necessary. Therefore, a total number of 1000 deaths (between four arms) will be used to trigger stopping of the study for the final analysis.

The primary efficacy end-point of time to all-cause mortality will be analysed using a Cox proportional hazards regression model allowing for important pre-defined covariates that may include, but not limited to, baseline FEV1, body mass index, geographical region and smoking status.

\section{DISCUSSION}

COPD often coexists with other conditions, in particular CVD. Importantly, the presence of one disease significantly affects the prognosis of the other. In this context, there is a need for evidence-based outcomes to support the efficacy of therapeutic modalities for the treatment of patients with COPD with concomitant CVD or its risk factors. As such, the SUMMIT trial aims to evaluate the impact of inhaled COPD therapy in patients with moderate COPD with, or at high risk for, CVD on all-cause mortality.

COPD and CVD may coexist for a number of reasons. A list of the most prominent mechanisms suggested is shown in table 3. Smoking is clearly an important shared risk factor and physical inactivity and deconditioning caused by COPD can clearly contribute to the risk of CVD. However, there may also be pathobiological links between the two diseases as a result of systemic inflammation that is common to both diseases. Inflammation in the small airways has been established as an initial event in the pathogenesis of COPD [21, 22] and its magnitude relates to the degree of airflow obstruction [22]. Recent studies suggest that the systemic inflammation seen in some patients with COPD may promote the development of atherosclerosis [23], the underlying cause of most ischaemic heart disease. In addition, systemic inflammation related to COPD (i.e. endothelial dysfunction) can produce structural changes (e.g. elastin fragmentation and degeneration, and increased collagen) and functional changes (e.g. reduced nitric oxide) in the wall of the aorta and medium-sized arteries resulting in decreased elasticity and increased stiffness [24]. Increased arterial stiffness is an independent predictor of all-cause and cardiovascular morbidity and mortality in hypertensive patients [25-27], as well as a predictor of cardiovascular events in general populations [28, 29].

The extent to which these types of data apply to patients with COPD is of increasing interest. ZUREIK et al. [30] demonstrated that carotid-femoral pulse wave velocity (PWV) was negatively associated with FEV1. Moreover, SABIT et al. [31] compared PWV in COPD patients with healthy smokers and ex-smokers who were free of cardiovascular disease and confirmed its negative relationship to FEV1. In addition, by multiple regression analysis, the circulating cytokine interleukin-6 was a strong predictor of PWV in this study. The large ECLIPSE (Evaluation of COPD Longitudinally to Identify Predictive Surrogate Endpoints) cohort study of patients with COPD showed that the presence of serum markers of inflammation increased the capacity to predict mortality compared with validated clinical predictors [32]. Increased PWV and elevated serum C-reactive protein (CRP) have also been demonstrated in patients with COPD compared with controls matched for age and smoking history [33]. These latter findings may be important as CRP is related to, and is a predictor of, cardiovascular risk [34]. Finally, in a cross-sectional study, MCALLISTER et al. [35] demonstrated that emphysema severity, as assessed by quantitative highresolution computerised tomography, is independently associated with arterial stiffness. These observations raise the potential of a possible link between the pulmonary and systemic inflammation observed in COPD, with impaired larger artery compliance as an important factor underlying the increased cardiovascular morbidity and mortality that has been observed

\begin{tabular}{|c|c|}
\hline TABLE 3 & $\begin{array}{l}\text { Potential mechanisms related to the presence } \\
\text { of cardiovascular disease in patients with chronic } \\
\text { obstructive pulmonary disease }\end{array}$ \\
\hline \multicolumn{2}{|c|}{ Systemic inflammation } \\
\hline \multicolumn{2}{|c|}{ Physical inactivity and deconditioning } \\
\hline \multicolumn{2}{|c|}{ Autonomic imbalance } \\
\hline \multicolumn{2}{|c|}{ Vascular endothelial dysfunction } \\
\hline \multicolumn{2}{|c|}{ Lower arterial compliance } \\
\hline \multicolumn{2}{|l|}{ Arrhythmias } \\
\hline \multicolumn{2}{|c|}{ Thrombogenicity } \\
\hline \multicolumn{2}{|c|}{$\begin{array}{l}\text { Increased afterload resulting from a greater fall in pleural pressure at each } \\
\text { inspiration due to impaired lung mechanics in chronic obstructive pulmonary } \\
\text { disease }\end{array}$} \\
\hline
\end{tabular}


in intervention studies $[10,14]$. Carotid-femoral PWV will be measured in a subset of patients in the SUMMIT trial at sites in the USA and the UK.

ICS have not been shown to reduce mortality in COPD. However, ICS do improve lung function and reduce exacerbations, both alone and in combination with a long-acting $\beta$-agonist [36, 37]. It is possible that the reduction in exacerbations in itself could result in a reduced risk of CVD events. MCALLISTER et al. [38] recently showed that patients admitted with a COPD exacerbation are likely to experience ischaemic events during this episode. In the EUROSCOP (European Respiratory Society Study on Chronic Obstructive Pulmonary Disease) study, long-term treatment with budesonide reduced ischaemic cardiac events in patients with mild COPD [39] without significantly affecting the risk of severe exacerbations. It is therefore likely that ICS in themselves may have benefits in patients with comorbid CVD.

The SUMMIT trial contains a placebo arm and this requires some consideration. All patients will be provided with a shortacting bronchodilator for relief and this is accordance with current COPD management strategies [1]. In addition, although potentially important, none of the tested medication classes in SUMMIT have been shown to provide long-term benefits to patients with COPD and this is an important gap in the evidence base. The SUMMIT trial is a long-term study in COPD and it may be prone to some of the biases observed in long-term trials in COPD resulting particularly from withdrawal [40]. However, using an event-driven design may reduce the risk of dilution bias. The study will use the same strict trial review as in the TORCH study including intensive follow-up and adjudication of deaths and CVD events. The SUMMIT study will be one of the largest multicentre, longterm COPD studies, and the first to investigate the effect of inhaled medications in patients with COPD and concomitant CVD. The data gathered may shed new light on the natural history of both these disorders.

\section{SUPPORT STATEMENT}

This study was funded by GlaxoSmithKline, London, UK.

\section{CLINICAL TRIAL}

This trial is registered at www.clinicaltrials.gov with identifier NCT01313676.

\section{STATEMENT OF INTEREST}

Conflict of interest information can be found alongside the online version of this article at www.erj.ersjournals.com

\section{ACKNOWLEDGEMENTS}

The authors' affiliations are as follows. J. Vestbo: Dept of Respiratory Medicine J, Odense University Hospital and University of Southern Denmark, Odense, Denmark, and Respiratory Research Group, Manchester Academic Health Sciences Centre, South Manchester University Hospital NHS Foundation Trust, Manchester, UK; J. Anderson: Research and Development, GlaxoSmithKline, Stockley Park, UK; R.D. Brook: Dept of Cardiology, University of Michigan Health System, Ann Arbor, MI, USA; P.M.A. Calverley: Dept of Medicine, Clinical Sciences Centre, University of Liverpool, University Hospital Aintree, Liverpool, UK; B.R. Celli: Pulmonary and Critical Care Division, Brigham and Women's Hospital, Harvard Medical School, Boston, MA, USA; C. Crim: Research and Development, GlaxoSmithKline, Research Triangle Park, NC, USA; B. Haumann:
Research and Development, GlaxoSmithKline, Stockley Park, UK; F.J. Martinez: Division of Pulmonary and Critical Care Medicine, University of Michigan Health System, Ann Arbor, MI, USA; J. Yates: Research and Development, GlaxoSmithKline, Research Triangle Park, NC, USA; D.E. Newby: Centre for Cardiovascular Science, University of Edinburgh, Edinburgh, UK.

The members of the Steering Committee are R.D. Brook, P.M.A. Calverley, B.R. Celli, F.J. Martinez, D. Newby, J. Vestbo (co-chair), J. Anderson, C. Crim, B. Haumann (co-chair) and J. Yates.

The members of the Safety and Efficacy Data Monitoring Committee are as follows: P. Lange (Chair; Section of Social Medicine, University of Copenhagen, Copenhagen, Denmark), R. Kay (RK Statistics Ltd, Great Longstone, UK), M. Dransfield (Division of Pulmonary, Allergy and Critical Care Medicine, University of Alabama, Birmingham, AL, USA), and S. Rajagopalan (DHLRI Vascular Research, Ohio State University Medical Center, Columbus, OH, USA).

The members of the End-point Committee are as follows: R. Wise (Chair; John Hopkins Asthma and Allergy Centre, Baltimore, MD, USA), D. Niewoehner (Pulmonary Section, VA Medical Center, Minneapolis, MN, USA), C. Gomez (Alabama Neurological Institute, Birmingham, AL, USA), S. Madger (Critical Care Division, Royal Victoria Hospital, Montreal, Canada), and M. Denvir (Centre for Cardiovascular Science, Queen's Medical Research Institute, Edinburgh, UK).

\section{REFERENCES}

1 Vestbo J, Hurd SS, Agusti AG, et al. Global Strategy for the diagnosis, management and prevention of chronic obstructive pulmonary disease: GOLD Executive Summary. Am J Respir Crit Care Med 2013; 187: 347-365.

2 Fabbri LM, Luppi F, Beghé B, et al. Complex chronic comorbidities of COPD. Eur Respir J 2008; 31: 204-212.

3 Mannino DM, Thorn D, Swensen A, et al. Prevalence and outcomes of diabetes, hypertension and cardiovascular disease in COPD. Eur Respir J 2008; 32: 962-969.

4 Agusti AG, Sauleda J, Miralles C, et al. Skeletal muscle apoptosis and weight loss in chronic obstructive pulmonary disease. Am J Respir Crit Care Med 2002; 166: 485-489.

5 Vestbo J, Prescott E, Almdal T, et al. Body mass, fat free body mass and prognosis in COPD patients from a random population sample. Am J Respir Crit Care Med 2006; 173: 79-83.

6 Bolton CE, Ionescu AA, Shiels KM, et al. Associated loss of fat-free mass and bone mineral density in chronic obstructive pulmonary disease. Am J Respir Crit Care Med 2004; 170: 1286-1293.

7 Tockman MS, Pearson JD, Fleg JL, et al. Rapid decline in FEV1: a new risk factor for coronary heart disease mortality. Am J Respir Crit Care Med 1995; 151: 390-398.

8 Weiss ST, Segal MR, Sparrow D, et al. Relation of FEV1 and peripheral blood leukocyte count to total mortality. The normative Aging Study. Am J Epidemiol 1995; 42: 493-498.

9 Hole DJ, Watt GCM, Davey-Smith G, et al. Impaired lung function and mortality risk in men and females: findings from the Renfrew and Paisley prospective population study. BMJ 1996; 313: 711-715.

10 Tashkin DP, Celli B, Senn S, et al. A 4-year trial of tiotropium in chronic obstructive pulmonary disease. N Engl J Med 2008; 359: 1543-1554.

11 Curkendall SM, DeLuise C, Jones JK, et al. Cardiovascular disease in patients with chronic obstructive pulmonary disease, Saskatchewan Canada cardiovascular disease in COPD. Am J Epidemiol 2006; 16: 63-70.

12 Anthonisen NR, Skeans MA, Wise RA, et al. The effects of a smoking cessation intervention on a 14.5-year mortality. Ann Intern Med 2005; 142: 233-239.

13 Mannino DM, Watt G, Hole D, et al. The natural history of chronic obstructive pulmonary disease. Eur Respir J 2006; 27: 627-643. 
14 Calverley PMA, Anderson JA, Celli B, et al. Salmeterol and fluticasone propionate and survival in chronic obstructive pulmonary disease. N Engl J Med 2007; 356: 775-789.

15 Sin DD, Man SFP. Why are patients with chronic obstructive pulmonary disease at increased risk of cardiovascular disease? The potential role of systemic inflammation in chronic obstructive pulmonary disease. Circulation 2003; 107: 1514-1519.

16 Watz H, Waschki B, Meyer T, et al. Physical activity in patients with COPD. Eur Respir J 2009; 33: 262-272.

17 Calverley PMA, Anderson JA, Celli B, et al. Cardiovascular events in patients with chronic obstructive pulmonary disease: TORCH study results. Thorax 2010; 65: 719-725.

18 Hankinson JL, Odencrantz JR, Fedan KB. Spirometric reference values from a sample of the general US population. Am J Respir Crit Care Med 1999; 159: 179-187.

19 Hankinson JL, Kawut SM, Shahar E, et al. Performance of American Thoracic Society recommended spirometry reference values in a multiethnic sample of adults. Chest 2010; 137: 138-145.

20 McGarvey LP, John M, Anderson JA, et al. Ascertainment of causespecific mortality in COPD: operations of the TORCH Clinical Endpoint Committee. Thorax 2007; 62: 411-415.

21 Hogg JC, Macklem PT, Thurlbeck WM. Site and nature of airway obstruction in chronic obstructive lung disease. N Engl J Med 1968; 278: 1355-1360.

22 Hogg JC, Chu F, Utokaparch S, et al. The nature of small-airway obstruction in chronic obstructive pulmonary disease. $N$ Engl J Med 2004; 350: 2645-2653.

23 Sevenoaks MJ, Stockley RA. Chronic obstructive pulmonary disease, inflammation and co-morbidity - a common inflammatory phenotype. Respir Res 2006; 7: 70.

24 Lakatta EG. Arterial and cardiac aging: major shareholders in cardiovascular disease enterprises. Part III: cellular and molecular clues to heart and arterial aging. Circulation 2003; 107: 490-497.

25 Laurent S, Boutouyrie $\mathrm{P}$, Asmar R, et al. Aortic stiffness is an independent predictor of all-cause mortality an cardiovascular mortality in hypertensive patients. Hypertension 2001; 37: 1236-1241.

26 Boutouyrie P, Tropeano AI, Asmar R, et al. Aortic stiffness is an independent predictor of primary coronary events in hypertensive patients; a longitudinal study. Hypertension 2002; 39: 10-15.

27 Laurent S, Katsahian S, Fassot C, et al. Aortic stiffness is an independent predictor of fatal stroke in essential hypertension. Stroke 2003; 34: 1203-1206.
28 Sutton-Tyrrell K, Najjar SS, Boudreau RM, et al. Elevated aortic pulse wave velocity, a marker of arterial stiffness, predicts cardiovascular events in well-functioning older adults. Circulation 2005; 111: 3384-3390.

29 Hansen TW, Staessen JA, Torp-Pedersen C, et al. Prognostic value of aortic pulse wave velocity as index of arterial stiffness in the general population. Circulation 2006; 113: 664-670.

30 Zureik M, Benetos A, Neukirch C, et al. Reduced pulmonary function is associated with central arterial stiffness in men. Am J Respir Crit Care Med 2001; 164: 2181-2185.

31 Sabit $\mathrm{R}$, Bolton $\mathrm{CE}$, Edwards $\mathrm{PH}$, et al. Arterial stiffness and osteoporosis in chronic obstructive pulmonary disease. Am J Respir Crit Care Med 2007; 175: 1259-1265.

32 Celli BR, Locantore N, Yates J, et al. Inflammatory biomarkers improve clinical prediction of mortality in chronic obstructive pulmonary disease. Am J Respir Crit Care Med 2012; 185: 1065-1072.

33 Mills NL, Miller JJ, Anand A, et al. Increased arterial stiffness in patients with chronic obstructive pulmonary disease: a mechanism for increased cardiovascular risk. Thorax 2008; 63: 306-311.

34 Ridker PM. High sensitivity C-reactive protein, inflammation, and cardiovascular risk: from concept to clinical practice to clinical benefit. Am Heart J 2004; 148: Suppl. 1, S19-S26.

35 McAllister DA, Maclay JD, Mills NL, et al. Arterial stiffness is independently associated with emphysema in patients with chronic obstructive pulmonary disease. Am J Respir Crit Care Med 2007; 176: 1208-1214.

36 Yang IA, Fong KM, Sim EH, et al. Inhaled corticosteroids for stable chronic obstructive pulmonary disease. Cochrane Database Syst Rev 2007; 2: CD002991.

37 Nannini LJ, Cates CJ, Lasserson TJ, et al. Combined corticosteroid and long-acting $\beta$-agonist in one inhaler versus long-acting $\beta$ agonists for chronic obstructive pulmonary disease. Cochrane Database Syst Rev 2007; 4: CD006829.

38 McAllister DA, Maclay JD, Mills NL, et al. Diagnosis of myocardial infarction following hospitalization for exacerbation of COPD. Eur Respir J 2012; 39: 1097-1103.

39 Löfdahl C-G, Postma DS, Pride NB, et al. Possible protection by inhaled budesonide against ischaemic cardiac events in mild COPD. Eur Respir J 2007; 29: 1115-1159.

40 Vestbo J, Anderson JA, Calverley PMA, et al. Bias due to withdrawal in long-term randomised trials in COPD: Evidence from the TORCH study. Clin Respir J 2011; 5: 44-49. 